\title{
A SIMPLE AND EFFICIENT SEGREGATED SMOOTHER FOR THE DISCRETE STOKES EQUATIONS*
}

\author{
FRANCISCO J. GASPAR ${ }^{\dagger}$, YVAN NOTAY $^{\ddagger}$, CORNELIS W. OOSTERLEE $^{\S}$, AND \\ CARMEN RODRIGO
}

\begin{abstract}
We consider the multigrid solution of the generalized Stokes equations with a segregated (i.e., equationwise) Gauss-Seidel smoother based on a Uzawa-type iteration. We analyze the smoother in the framework of local Fourier analysis, and obtain an analytic bound on the smoothing factor showing uniform performance for a family of Stokes problems. These results are confirmed by the numerical computation of the two-grid convergence factor for different types of grids and discretizations. Numerical results also show that the actual convergence of the W-cycle is approximately the same as that obtained by a Vanka smoother, despite this latter smoother being significantly more costly per iteration step.
\end{abstract}

Key words. multigrid methods, generalized Stokes equations, Uzawa smoother, smoothing theory, local Fourier analysis, staggered grids, nonstaggered grids

AMS subject classifications. $65 \mathrm{~N} 55,65 \mathrm{~F} 10$

DOI. $10.1137 / 130920630$

1. Introduction. The need to solve the generalized Stokes equations appears in incompressible fluid dynamics and in some structural mechanics applications, as in plasticity, beam and shell studies. In the simulation of fluid flow by the nonlinear time-dependent Navier-Stokes equations, a generalized Stokes problem also has to be solved at each nonlinear iteration.

The Stokes equations form a saddlepoint problem, and depending on the choice of discretization method, one may end up with a matrix in $2 \times 2$ block form, in which the lower diagonal block is either a zero block or a block containing very small matrix elements. Saddlepoint problems are well known and well studied in numerical analysis. An overview of this topic has been presented in [2].

The tradition of solving Stokes equations with multigrid is long, and interesting approaches have been presented during the last 35 years. Basically two approaches have established themselves, and their difference lies in the type of smoothing operator adopted. A state-of-the-art smoother for the Stokes equations is a Gauss-Seideltype coupled Vanka smoother [18], in which the primary unknowns, pressure and the velocities in a grid cell, are updated simultaneously. Each smoothing step then requires us to solve as many small systems as there are cells in the grid.

*Submitted to the journal's Methods and Algorithms for Scientific Computing section May 10, 2013; accepted for publication (in revised form) March 31, 2014; published electronically June 12, 2014. This work was supported by the Spanish project FEDER/MCYT MTM2010-16917, the DGA (Grupo consolidado PDIE), and the CWI MAC2 budget.

http://www.siam.org/journals/sisc/36-3/92063.html

$\dagger$ Department of Applied Mathematics, University of Zaragoza, Pedro Cerbuna 9, 50012, Zaragoza, Spain (fjgaspar@unizar.es, unizar.es/pde/fjgaspar/index.html).

$\ddagger$ Université Libre de Bruxelles, Service de Métrologie Nucléaire (C.P. 165-84), 50 Av. F.D. Roosevelt, B-1050 Brussels, Belgium (ynotay@ulb.ac.be, homepages.ulb.ac.be/ ynotay). Yvan Notay is Research Director of the Fonds de la Recherche Scientifique - FNRS.

$\S$ CWI, Centrum Wiskunde \& Informatica, Science Park 123, 1098 XG Amsterdam, the Netherlands, and Delft University of Technology, the Netherlands (c.w.oosterlee@cwi.nl, ta.twi.tudelft.nl/ $\mathrm{mf} /$ users/oosterle).

『 Department of Applied Mathematics, University of Zaragoza, María de Luna 3, 50018, Zaragoza, Spain (carmenr@unizar.escarmenr@unizar.es). 
Decoupled, i.e., equationwise, smoothing is often preferred for reasons of cost efficiency. For Stokes problems, one first transforms the discrete system such that equationwise smoothing on the transformed discrete system brings excellent smoothing factors. Equationwise, decoupled smoothing on a transformed system is called distributive smoothing $[4,22]$.

These two state-of-the-art smoothers are somewhat involved, as compared to basic smoothing techniques for scalar elliptic PDEs. Straightforward generalization of the basic pointwise smoothing principle to systems of equations, like the Stokes equations, implies a segregated, decoupled smoothing scheme in which the individual equations of the PDE system are considered to be scalar equations. In this paper we present such a smoother. Issues associated with standard equationwise smoothing procedures like damped Jacobi and Gauss-Seidel are avoided by considering an Uzawa-type iteration. A similar Uzawa smoother was presented in a Ph.D. thesis by P. Nigon, as well as in a conference proceedings paper in the 1980s [13]. Here we present a new analysis of this approach leading to a deeper understanding of the multigrid performance and to the formulation of a more efficient variant.

For the velocity components, any basic smoothing technique applicable to scalar elliptic PDEs is possible, but our analysis favors symmetric schemes. This leads us to focus on symmetric Gauss-Seidel smoothing; that is, one forward pointwise GaussSeidel sweep for all velocity unknowns followed by one backward sweep. In [13], two forward Gauss-Seidel sweeps were used instead, and numerical results reveal that, everything else being equal, our choice is indeed more effective.

The Uzawa-like procedure amounts to a simple Richardson iteration for the smoothing of the pressure unknowns. As usual, such iteration involves a relaxation, or damping, parameter.

Here we provide an upper bound on the smoothing factor [17] associated with the proposed Uzawa smoother that indicates how the different parameters may affect the convergence, and we deduce a rule of thumb to select the aforementioned damping parameter as a function of the main problem characteristics.

We then consider three typical discretizations of a family of Stokes problems, ranging from stationary to time dependent with a small time step. In each case, we perform numerical experiments that confirm the relevance of our analysis and the efficiency of the proposed approach. Our upper bound on the smoothing factor gives an excellent estimation of the exact smoothing factor, and selecting the parameter according to the proposed rule of thumb is indeed optimal or near optimal. The smoothing factor reflects well the two-grid convergence factor computed by local Fourier analysis (LFA), as well as the actual convergence factor associated with practical multigrid cycles. Finally, the convergence is roughly the same as that obtained with the Vanka smoother; since this latter is significantly more costly per step, it means that our approach is effective, at least for the selected examples.

The outline of the paper is as follows. In section 2, we present the generalized (i.e., parametrized) Stokes equations and give properties of the linear systems resulting from their discretizations. The Uzawa smoother is introduced in section 3, where we also develop our analysis of the associated smoothing factor. This analysis is then numerically illustrated in the subsequent sections, where we consider successively a staggered marker-and-cell discretization (section 4), a collocated grid discretization with an artificial pressure term (section 5), and a stabilized linear finite element method on an equilateral triangular mesh (section 6). Concluding remarks are given in section 7 . 
2. The generalized Stokes equations and their discretization. Given a bounded polygonal domain $\Omega \subseteq \mathbb{R}^{2}$ with a Lipschitz-continuous boundary $\partial \Omega$, the generalized Stokes problem in $d$ dimensions (with $d=2$ or $d=3$ ) requires finding the velocity vector $\mathbf{u}: \Omega \rightarrow \mathbb{R}^{d}$, and the kinematic pressure field $p: \Omega \rightarrow \mathbb{R}$, satisfying

$$
\begin{array}{rlrl}
\xi \mathbf{u}-\nu \Delta \mathbf{u}+\nabla p=\mathbf{f} & \text { in } \Omega, \\
\nabla \cdot \mathbf{u}=0 & & \text { in } \Omega,
\end{array}
$$

where $\mathbf{f}$ represents a prescribed force, and the parameters $\nu>0$ (viscosity) and $\xi \geq 0$ are given. The latter is often a quantity proportional to the inverse of the time step in an implicit time integration method applied to a nonstationary Stokes problem; $\xi=0$ corresponds to the classical stationary Stokes problem. Whatever the chosen scheme, the discretization of (2.1) leads to a linear system of the form

$$
K=\left(\begin{array}{cc}
A & B^{T} \\
B & -C
\end{array}\right) .
$$

In this matrix, $A$ is the discrete representation of the operator $\xi-\nu \Delta$; more precisely, $A$ is block diagonal with one diagonal block per spatial dimension, being the discrete operator acting on one of the velocity components. It further follows that $A$ is symmetric positive definite (SPD). The matrix block $B^{T}$ is the discrete gradient and $B$ the discrete divergence; $C$ is a stabilization term which is needed by some discretization schemes to avoid spurious solutions. Such spurious solutions arise when the discrete gradient admits more than the constant vector in its null space or near null space; i.e., when the discrete gradient is zero or near zero for some spurious pressure modes. The existence of such modes depends on which discretization scheme is used for velocities and pressure. We refer to, e.g., [20] and [7] for more details on, respectively, finite difference and finite element discretizations. Note a required property of the stabilization operator: if $B$ is not full rank, $C$ has to be positive definite on the null space of $B^{T}$, which further entails that the system matrix is nonsingular [2].

An important exception to this latter rule is when the boundary conditions are such that the physical pressure is only determined up to a constant. To make the problem well-posed, one needs then to impose some additional condition, such as

$$
\int_{\Omega} p \mathrm{~d} \mathbf{x}=0
$$

At the discrete level, this is reflected in the fact that $B^{T} \mathbf{1}=\mathbf{0}$, where $\mathbf{1}$ is the vector of all ones. Hence, matrix $K$ may be singular with a null space spanned by $\left(\begin{array}{lll}\mathbf{0} & \mathbf{1}^{T}\end{array}\right)^{T}$. Whether $K$ is singular or not depends on $C$, but, to preserve accuracy, stabilization operators need to be small for smooth modes, and often satisfy $C \mathbf{1}=0$ as well (see section 5 below for an example).

The singularity of $K$ poses no particular difficulties when solving the linear system by an iterative method; see, e.g., [7, section 8.3] for a detailed discussion in the context of the Navier-Stokes equations. Basically, the system is compatible because the righthand side of (2.1) together with the lack of boundary conditions for the pressure entail that the right-hand side of the discrete system is zero for the second block of equations. Further, starting from, say, the zero vector as an initial approximation, all updates are kept orthogonal to the kernel vector $\left(\begin{array}{ll}\mathbf{0} & \mathbf{1}^{T}\end{array}\right)^{T}$; that is, all updates are such that the discrete pressure unknowns have mean value zero, implying that the constraint (2.3) is satisfied at the discrete level by all successive approximations. Regarding the iteration 
matrix, it means that the eigenvalue 1 associated with the singular mode does not have to be taken into account. The convergence is governed by the "effective" spectral radius, which corresponds to the maximum in modulus of the other eigenvalues.

Considering multigrid solution methods in particular, prolongation operators are often such that a discrete pressure with mean value zero on the coarse grid maintains mean value zero once prolongated to the fine grid; i.e., no special treatment is needed when the coarse solver picks up the "correct" solution. The situation may be different regarding relaxation. However, as will be seen in the next section, the smoother that we propose in this work preserves the mean value of the discrete pressure as soon as $B^{T} \mathbf{1}=\mathbf{0}$ and $C \mathbf{1}=\mathbf{0}$; i.e., here too, no particular treatment is needed to enforce (2.3) at the discrete level when the system is singular.

3. The Uzawa smoother. For the Stokes operator, current state-of-the-art smoothers are Vanka and distributive relaxations, as mentioned in the introduction. Both ensure a form of coupling between the relaxation processes applied to the unknowns belonging to a same grid cell. Interestingly, the smoother proposed here does not fall into these two categories of relaxation methods, and is truly decoupled.

Our approach is not rooted in multigrid research, but in the Uzawa method (e.g., [2, section 8.1]), which is an iterative scheme to solve linear systems with a matrix of the form (2.2). It amounts to performing stationary iterations with the preconditioner

$$
\left(\begin{array}{cc}
A & \\
B & -\omega^{-1} I
\end{array}\right)
$$

where $\omega$ is some positive parameter. The presence of the matrix block $A$ in this preconditioner implies an exact solve for velocity at each iteration. This makes the approach costly, and the Uzawa method is in fact popular thanks to the "inexact variants" that replace this block by some preconditioner for $A$.

In the multigrid context, it seems natural to consider as smoother this operator where $A$ is replaced by a typical smoother $M_{A}$; that is, to consider the smoother

$$
M=\left(\begin{array}{cc}
M_{A} & 0 \\
B & -\omega^{-1} I
\end{array}\right) .
$$

Because of the structure of $A$ (block diagonal with diagonal blocks corresponding to discrete $\xi-\nu \Delta$ ), all commonly used smoothers for scalar elliptic PDEs can be used to define $M_{A}$.

The smoother (3.1) is a decoupled smoother, and a single smoothing step can be described as follows: given $\left(\mathbf{u}^{T}, \mathbf{p}^{T}\right)^{T}$ an approximation of the solution to the system, compute the relaxed approximation $\left(\widehat{\mathbf{u}}^{T}, \widehat{\mathbf{p}}^{T}\right)^{T}$ according to

- apply smoother $M_{A}$ to relax the system $A \mathbf{u}=\mathbf{f}-B^{T} \mathbf{p}$; i.e., $\hat{\mathbf{u}}=\mathbf{u}+M_{A}^{-1}\left(\mathbf{f}-A \mathbf{u}-B^{T} \mathbf{p}\right)$;

- update the pressure: $\hat{\mathbf{p}}=\mathbf{p}+\omega(B \hat{\mathbf{u}}-C \mathbf{p})$.

When $K$ is singular because $B^{T} \mathbf{1}=\mathbf{0}$ and $C \mathbf{1}=\mathbf{0}$, one has $\mathbf{1}^{T} B=\mathbf{1}^{T} C=0$ and therefore $\mathbf{1}^{T} \hat{\mathbf{p}}=\mathbf{1}^{T} \mathbf{p}$, i.e., the relaxation associated with the Uzawa smoother preserves the mean value of the discrete pressure as discussed at the end of section 2 .

When $M_{A}$ consists of two forward Gauss-Seidel sweeps for velocities, this scheme is the one suggested in [13]. In this work, we shall consider another variant, where $M_{A}$ is based on symmetric Gauss-Seidel iterations for $A$, i.e.,

$$
M_{A}=\left(D_{A}+L_{A}\right) D_{A}^{-1}\left(D_{A}+U_{A}\right)
$$


where $D_{A}, L_{A}$, and $U_{A}$ are, respectively, the diagonal, the strict lower, and the strict upper parts of $A$. Besides being a good smoother, such an $M_{A}$ satisfies two important properties needed by our theoretical analysis. First, it is SPD when $A$ is SPD and, next, the associated largest eigenvalue satisfies (see, e.g., [1, Theorem 7.17])

$$
\lambda_{\max }\left(M_{A}^{-1} A\right) \leq 1 .
$$

Numerical experiments will also reveal that the convergence associated with such an $M_{A}$ is often faster than that obtained by two SOR sweeps as in [13], for essentially the same cost.

Our analysis of the Uzawa smoother requires us to first make the assumptions and simplifications associated with the LFA framework. LFA (also known as local mode analysis) is the most powerful tool for the quantitative analysis and design of efficient multigrid methods. In this analysis an infinite regular grid is considered and boundary conditions are not taken into account. LFA was introduced by Brandt in [3] and afterwards extended in [5]. A good introduction can be found in the paper by Stüben and Trottenberg [16] and in the books by Wesseling [19], Trottenberg, Oosterlee, and A. Schüller [17], and Wienands and Joppich [21].

In this framework, the discrete operators can be expressed in a basis (the Fourier basis) in which they have a simpler form. For instance, discrete representations of $\xi-\nu \Delta$ are typically diagonal, each component corresponding to a particular "frequency," LFA is further based on the classification of these frequencies in "low frequency" components and "high frequency" components. The idea behind this is the following: multigrid methods work if the smoother and the coarse grid operator interact properly, and it is generally expected that the smoother damps the high frequency components of the error whereas the coarse grid correction damps the low frequency ones. Hence, regarding the smoother, most important is its action on the high frequency components.

It is convenient to permute the components so that the high frequency ones are labeled first. Then $A$, expressed in the Fourier basis, admits the block diagonal form

$$
A_{\mathcal{F}}=\left(\begin{array}{cc}
A_{\text {high }} & \\
& A_{\text {low }}
\end{array}\right) \text {. }
$$

The exact structure of $B$ in this basis depends on the discretization scheme, but, as a general rule, the different types of frequency do not mismatch. That is, the discrete gradient of a low (resp., high) frequency pressure vector involve only low (resp., high) frequency velocity components. Moreover, typical stabilization matrices $C$ are also block diagonal with respect to this partitioning of frequencies. Hence, the system matrix in the Fourier basis has the form

$$
K_{\mathcal{F}}=\left(\begin{array}{cccc}
A_{\text {high }} & & B_{\text {high }}^{*} & \\
& A_{\text {low }} & & B_{\text {low }}^{*} \\
B_{\text {high }} & & -C_{\text {high }} & \\
& B_{\text {low }} & & -C_{\text {low }}
\end{array}\right) .
$$

For $B_{\text {high }}$ and $B_{\text {low }}$, the conjugate transpose appears in the top right block because the basis transformation is orthogonal but in general complex. Hence the transformed matrix is no longer real, and blocks that were transposes of each other become conjugate transposes of each other. Blocks that were symmetric become Hermitian, and remain definite if they were previously definite. In particular, if $C$ was positive definite on the null space of $B^{*}, C_{h i g h}$ is positive definite on the null space of $B_{\text {high }}^{*}$, and $C_{\text {low }}$ is positive definite on the null space of $B_{l o w}^{*}$. 
The smoother has to be expressed in the same basis. In fact, standard smoothers for scalar elliptic PDEs are also diagonal when expressed in the Fourier basis; hence, applying to $M_{A}$ the same transformations and permutations as to $A$ yields

$$
M_{\mathcal{F}}^{(A)}=\left(\begin{array}{ll}
M_{\text {high }}^{(A)} & \\
& M_{\text {low }}^{(A)}
\end{array}\right) .
$$

Because the basis transformation is orthogonal, the identity remains the identity in the new basis. Hence the smoother (3.1) becomes

$$
M_{\mathcal{F}}=\left(\begin{array}{cccc}
M_{\text {high }}^{(A)} & & & \\
& M_{\text {low }}^{(A)} & & \\
B_{\text {high }} & & -\omega^{-1} I & \\
& B_{\text {low }} & & -\omega^{-1} I
\end{array}\right) .
$$

Now, despite the simpler form of the operators in the new basis, performing a complete analysis of the multigrid iteration matrix often remains out of reach for systems of PDEs. If one is interested in the smoother's performance, insight can be gained by considering a simplified (and, in some sense, idealized) scheme, in which the coarse grid corrections erase exactly all low frequency components of the error, while leaving the high frequency ones unchanged [17]. The corresponding convergence factor is referred to as the smoothing factor, and the following theorem allows us to bound this latter for the Uzawa smoother (3.1).

Theorem 3.1. Let $K_{\mathcal{F}}$ be defined by (3.4), where $A_{\text {high }}, A_{\text {low }}, C_{\text {high }}$, and $C_{\text {low }}$ are, respectively, $n_{\text {high }} \times n_{\text {high }}, n_{\text {low }} \times n_{\text {low }}, m_{\text {high }} \times m_{\text {high }}$, and $m_{\text {low }} \times m_{\text {low }}$ Hermitian matrices with $m_{\text {high }} \leq n_{\text {high }}$. Assume that $A_{\text {high }}$ is positive definite, that $C_{\text {high }}$ is nonnegative definite, and that either $B_{\text {high }}$ has full rank or $C_{\text {high }}$ is positive definite on the null space of $B_{\text {high }}^{*}$.

Let $M_{\mathcal{F}}$ be defined by (3.5), where $M_{\text {high }}^{(A)}, M_{\text {low }}^{(A)}$ are, respectively, $n_{\text {high }} \times n_{\text {high }}$ and $n_{\text {low }} \times n_{\text {low }}$ Hermitian positive definite matrices.

Define the smoothing factor $\mu$ by

$$
\mu=\rho\left(\left(I-M_{\mathcal{F}}^{-1} K_{\mathcal{F}}\right) T_{c}^{(\mathcal{F})}\right),
$$

where

$$
\begin{aligned}
& T_{c}^{(\mathcal{F})}=\left(\begin{array}{cccc}
I_{n_{\text {high }}} & & & \\
& 0_{n_{\text {low }} \times n_{\text {low }}} & & \\
& & I_{m_{\text {high }}} & \\
& & 0_{m_{\text {low }} \times m_{\text {low }}}
\end{array}\right) . \\
& \text { If } \lambda_{\max }\left(M_{\text {high }}^{(A)}{ }^{-1} A_{\text {high }}\right) \leq 1 \text {, letting } \\
& \mu_{A}=\rho\left(I-M_{\text {high }}^{(A)}{ }^{-1} A_{h i g h}\right)
\end{aligned}
$$

and

$$
\mu_{S}=\rho\left(I-\omega\left(C_{\text {high }}+B_{\text {high }} A_{\text {high }}^{-1} B_{\text {high }}^{*}\right)\right)
$$

it follows that

$$
\mu \leq \bar{\mu}=\max \left(\left(\mu_{A}\right)^{1 / 2}, \mu_{S}\right) .
$$


Proof. Using

$$
M_{\mathcal{F}}^{-1}=\left(\begin{array}{cccc}
M_{\text {high }}^{(A)}{ }^{-1} & & \\
\omega B_{\text {high }} M_{\text {high }}^{(A)}{ }^{-1} & M_{\text {low }}^{(A)^{-1}} & \\
& \omega B_{\text {low }} M_{\text {low }}^{(A)^{-1}} & -\omega I_{m_{\text {high }}} & \\
& & -\omega I_{m_{\text {low }}}
\end{array}\right),
$$

one can check that

$$
\mu=\rho\left(I-M_{\text {high }}^{-1} K_{\text {high }}\right)
$$

where

$$
K_{\text {high }}=\left(\begin{array}{cc}
A_{\text {high }} & B_{h i g h}^{*} \\
B_{\text {high }} & -C_{\text {high }}
\end{array}\right), \quad M_{\text {high }}=\left(\begin{array}{cc}
M_{\text {high }}^{(A)} & \\
B_{h i g h} & -\omega^{-1} I_{m_{\text {high }}}
\end{array}\right)
$$

The required result then follows from Corollary 4.5 in [14]. Strictly speaking, this latter result is proved only for real matrices. But, examining thoroughly its proof as well as that of the theorems it is based on, it turns out that the extension to the complex case runs smoothly, reading "symmetric" as "Hermitian" and "transpose" as "conjugate transpose."

Note that $(3.3)$ implies $\lambda_{\max }\left(M_{\text {high }}^{(A)}{ }^{-1} A_{\text {high }}\right) \leq 1$. Hence the only additional assumption in Theorem 3.1 always holds with the symmetric Gauss-Seidel smoother. This theorem provides a bound on the smoothing factor involving only the smoothing factor $\mu_{A}$ associated with the smoother for velocities, and $\mu_{S}$, which can be seen as the smoothing factor associated with Richardson iterations for the Schur complement

$$
S=C+B A^{-1} B^{T} .
$$

Bounding $\mu_{A}$ raises no particular difficulties. Often one can reuse available results for scalar elliptic PDEs $[17,21]$. The analysis of $\mu_{S}$ is more tricky. Theorem 3.2, below, is helpful in this respect. As will be seen, it helps to select $\omega$ in a sensible way. It allows us to estimate $\mu_{S}$ as a function of the main parameters associated with a discrete representation of the problem (2.1). In this context, $A=A^{(0)}+\nu G$, with $A^{(0)}$ a discrete representation of $(-\Delta)$ and $G$ of the identity. Further, $C=\nu^{-1} C^{(0)}$, where $C^{(0)}$ is the proper stabilization term when $\nu=1 .{ }^{1}$ Representing these matrices in the Fourier basis and splitting them into high and low frequency components then naturally leads to the representation (3.8) the theorem is based on.

TheOREM 3.2. Let the assumptions of Theorem 3.1 hold. Let $\nu>0$ and $\xi \geq 0$ be real numbers such that

$$
A_{h i g h}=\nu A_{\text {high }}^{(0)}+\xi G_{h i g h} \quad \text { and } \quad C_{h i g h}=\nu^{-1} C_{\text {high }}^{(0)}
$$

for some Hermitian positive definite matrices $A_{\text {high }}^{(0)}, G_{\text {high }}$, and Hermitian nonnegative definite matrix $C_{\text {high }}^{(0)}$. Let $h>0, \beta>0, \eta>0$, and $\gamma \geq 0$ be real numbers such

\footnotetext{
${ }^{1}$ Because the quality of the stabilization depends on the eigenvalue distribution of the Schur complement [7], it is clear that the stabilization term has to be kept proportional to $\nu^{-1}$.
} 
that

$$
\begin{aligned}
\lambda_{\max }\left(C_{\text {high }}+B_{\text {high }} A_{\text {high }}^{(0)}{ }^{-1} B_{\text {high }}^{*}\right) & \leq \beta, \\
\lambda_{\max }\left(G_{\text {high }}^{-1} A_{\text {high }}^{(0)}\right) & \leq \frac{1}{\eta h^{2}}, \\
\lambda_{\max }\left(C_{\text {high }}^{(0)}\right) & \leq \gamma .
\end{aligned}
$$

Then, setting, for some positive real number $\tau$,

$$
\omega=\frac{\tau \nu\left(1+\eta \frac{\xi h^{2}}{\nu}\right)}{\beta+\gamma \eta \frac{\xi h^{2}}{\nu}},
$$

one has $\mu_{S}<1$ if $\tau<2$. Moreover, letting

$$
\begin{aligned}
\kappa_{\beta} & =\frac{\beta}{\lambda_{\min }\left(C_{\text {high }}+B_{\text {high }} A_{\text {high }}^{(0)}{ }^{-1} B_{\text {high }}^{*}\right)}, \\
\kappa_{\eta} & =\frac{1}{\eta h^{2} \lambda_{\min }\left(G_{\text {high }}^{-1} A_{\text {high }}^{(0)}\right)}, \\
\kappa_{\gamma} & = \begin{cases}1 & \text { if } C_{\text {high }}=0, \\
\frac{\gamma}{\lambda_{\min }\left(C_{\text {high }}^{(0)}\right)} & \text { if } C_{\text {high }} \text { is positive definite, } \\
\infty & \text { otherwise, },\end{cases}
\end{aligned}
$$

we obtain

$$
\begin{aligned}
\mu_{S} & \leq \max \left(\tau-1,1-\tau \frac{1+\eta \frac{\xi h^{2}}{\nu}}{1+\kappa_{\eta} \eta \frac{\xi h^{2}}{\nu}} \frac{\frac{\beta}{\kappa_{\beta}}+\frac{\gamma}{\kappa_{\gamma}} \kappa_{\eta} \eta \frac{\xi h^{2}}{\nu}}{\beta+\gamma \eta \frac{\xi h^{2}}{\nu}}\right) \\
& \leq \max \left(\tau-1,1-\frac{\tau}{\max \left(\kappa_{\beta} \kappa_{\eta}, \kappa_{\gamma}\right)}\right) .
\end{aligned}
$$

In particular, if $\xi=0$,

$$
\mu_{S} \leq \max \left(\tau-1,1-\frac{\tau}{\kappa_{\beta}}\right) .
$$

Proof. Reading inequalities in the nonnegative definite sense $(Q \geq R$ if and only if $Q-R$ is nonnegative definite), one first checks that

$$
\begin{aligned}
C_{\text {high }}+B_{\text {high }} A_{\text {high }}^{-1} B_{\text {high }}^{*} & =\nu^{-1} C_{\text {high }}^{(0)}+B_{\text {high }}\left(\nu A_{\text {high }}^{(0)}+\xi G_{\text {high }}\right)^{-1} B_{\text {high }}^{*} \\
& \leq \nu^{-1}\left(C_{\text {high }}^{(0)}+B_{h i g h}\left(1+\frac{\eta \xi h^{2}}{\nu}\right)^{-1} A_{\text {high }}^{(0)}{ }^{-1} B_{h i g h}^{*}\right) \\
& =\frac{\nu^{-1}}{1+\eta \frac{\xi h^{2}}{\nu}}\left(C_{\text {high }}^{(0)}+B_{\text {high }} A_{\text {high }}^{(0)}{ }^{-1} B_{\text {high }}^{*}+\eta \frac{\xi h^{2}}{\nu} C_{\text {high }}^{(0)}\right) \\
& \leq \frac{\nu^{-1}}{1+\eta \frac{\xi h^{2}}{\nu}}\left(\beta+\gamma \eta \frac{\xi h^{2}}{\nu}\right) I .
\end{aligned}
$$


Hence, with $\omega$ given by (3.12), all eigenvalues of $\omega\left(C_{\text {high }}+B_{\text {high }} A_{\text {high }}^{-1} B_{\text {high }}^{*}\right)$ are in the interval $(0, \tau)$, entailing $\mu_{S}<1$ if $0<\tau<2$. Moreover,

$$
\mu_{S} \leq \max \left(\tau-1,1-\frac{\tau \nu\left(1+\eta \frac{\xi h^{2}}{\nu}\right) \lambda_{\min }\left(C_{h i g h}+B_{h i g h} A_{h i g h}^{-1} B_{h i g h}^{*}\right)}{\beta+\gamma \eta \frac{\xi h^{2}}{\nu}}\right),
$$

whereas, reusing (3.19),

$$
\begin{aligned}
C_{h i g h}+B_{h i g h} A_{\text {high }}^{-1} B_{\text {high }}^{*} & \geq \nu^{-1}\left(C_{\text {high }}^{(0)}+B_{\text {high }}\left(1+\kappa_{\eta} \eta \frac{\xi h^{2}}{\nu}\right)^{-1} A_{\text {high }}^{(0)}{ }^{-1} B_{h i g h}^{*}\right) \\
& =\frac{\nu^{-1}}{1+\kappa_{\eta} \eta \frac{\xi h^{2}}{\nu}}\left(C_{\text {high }}^{(0)}+B_{h i g h} A_{\text {high }}^{(0)}{ }^{-1} B_{h i g h}^{*}+\kappa_{\eta} \eta \frac{\xi h^{2}}{\nu} C_{h i g h}^{(0)}\right) \\
& \geq \frac{\nu^{-1}}{1+\kappa_{\eta} \eta \frac{\xi h^{2}}{\nu}}\left(\frac{\beta}{\kappa_{\beta}}+\frac{\gamma}{\kappa_{\gamma}} \kappa_{\eta} \eta \frac{\xi h^{2}}{\nu}\right) I .
\end{aligned}
$$

Then (3.16) and (3.18) follow by considering, respectively, the second and the first term in the maximum. Eventually, (3.17) follows from (3.16) using

$$
\frac{1+\eta \frac{\xi h^{2}}{\nu}}{1+\kappa_{\eta} \eta \frac{\xi h^{2}}{\nu}} \geq \frac{1}{\kappa_{\eta}} \quad \text { and } \quad \frac{\frac{\beta}{\kappa_{\beta}}+\frac{\gamma}{\kappa_{\gamma}} \kappa_{\eta} \eta \frac{\xi h^{2}}{\nu}}{\beta+\gamma \eta \frac{\xi h^{2}}{\nu}} \geq \min \left(\frac{1}{\kappa_{\beta}}, \frac{\kappa_{\eta}}{\kappa_{\gamma}}\right) \text {. }
$$

Because of the different quantities involved, the insight gained from this theorem may seem unclear. How to use the above results is best seen by examples, hence we primarily refer the reader to the following sections where typical applications are considered.

A few general remarks can be made. First of all, only estimates of $\beta, \gamma$, and $\eta$ are needed to use the rule (3.12) which we suggest for practical implementations; $\kappa_{\beta}, \kappa_{\eta}$, and $\kappa_{\gamma}$ are less critical because their values only influence the bounds on $\mu_{S}$ and not the practical usage of the method. Regarding these latter parameters, it is sufficient to know that they are bounded independently of the mesh size and possibly other problem parameters, so that the bounds (3.16), (3.17), (3.18) remain significantly below one.

Equation (3.12) also involves the additional parameter $\tau$, and one would like to use the optimal value, which does not seem possible having only estimates for $\beta, \gamma$, and $\eta$. In fact, the presentation followed in Theorem 3.2 is motivated by the observation that, at least in the examples considered, the numerical results obtained following (3.12) are fairly insensitive to $\tau$ (see the next section). We explain this with the bound (3.6) of Theorem 3.1: as soon as $\mu_{S} \leq\left(\mu_{A}\right)^{1 / 2}$, fine tuning to further decrease $\mu_{S}$ does not influence the final bound on the smoothing factor. In the next sections, we therefore mostly display results obtained by fixing $\tau=1.4$, which we found optimal or close to optimal in all cases. This value is close to the largest one such that $\tau-1$ is still below $\left(\mu_{A}\right)^{1 / 2}$ for common values of this parameter. Thus, in some sense, it minimizes the second term of the maximum expressions in (3.16), (3.17), (3.18) under the constraint that the first term remains below $\left(\mu_{A}\right)^{1 / 2}$.

Considering (3.12), it is also clear that only $\beta$ is needed when $\xi=0$, and, more generally (given that $\gamma \leq \beta$ by virtue of (3.9), (3.11)), for any $\xi$ such that $\eta \frac{\xi h^{2}}{\nu} \ll 1$. When $\eta$ is smaller than 1 , as in all examples discussed below, this in particular holds when $\frac{\xi h^{2}}{\nu} \ll 1$; that is, in most common situations. 
Two approaches can be followed to estimate the constants $\beta, \eta$, and $\gamma$. If one performs an LFA for the problem at hand (as we do in the next sections), the matrices involved in the definitions (3.9), (3.10) and (3.11) are explicitly available, and, in general, diagonal. Deriving their largest eigenvalues is a straightforward task.

Alternatively, let $A^{(0)}, G$, and $C^{(0)}$ be discrete representations of, respectively, $(-\Delta)$, the identity, and the stabilization term for $\nu=1$; i.e., the matrices from where $A_{\text {high }}^{(0)}, G_{\text {high }}$, and $C_{\text {high }}^{(0)}$ are derived. Clearly, (3.9), (3.10), (3.11) a fortiori hold if

$$
\begin{aligned}
\lambda_{\max }\left(C^{(0)}+B A^{(0)^{-1}} B^{T}\right) & \leq \beta, \\
\lambda_{\max }\left(G^{-1} A^{(0)}\right) & \leq \frac{1}{\eta h^{2}}, \\
\lambda_{\max }\left(C^{(0)}\right) & \leq \gamma ;
\end{aligned}
$$

that is, if the inequalities hold with respect to the complete matrices and not just with respect to their restrictions to the high frequency components. Hence one may obtain valid estimates for $\beta, \eta$, and $\gamma$ by analyzing these latter inequalities instead of (3.9), (3.10), (3.11). This is attractive if one does not want to perform a full LFA of the problem: the inequalities (3.20), (3.21), and (3.22) do not depend on whether one uses the Fourier basis or the matrices from the discretization. This may give some loss of accuracy and, for instance, using $\beta$ larger than the smallest value satisfying (3.9) leads to an underestimation of $\omega$ in (3.12). However, in the examples considered below, we found that this approach gave only minor differences in the problem parameters $\beta, \eta$, and $\gamma$, and hardly any impact on the method's performance.

Taking this latter viewpoint also allows us to quickly estimate the order of magnitude of the different constants. Here we have to distinguish finite difference and finite element discretizations.

In the finite difference case, it turns out that $\beta$ is a constant close to one, whereas, since $G$ is the identity, (3.21) holds with $\eta=1 / 8$ in two-dimensional cases ( $A$ is five point Laplacian) and $\eta=1 / 12$ in three dimensions ( $A$ is seven point Laplacian). The parameter $\gamma$ is equal to zero when a stable scheme is used, and depends on the stabilization term otherwise; see section 5 for an example. Note that if $\gamma$ does not vanish, it has to be a constant smaller or equal to $\beta$ (compare (3.9) and (3.11)).

For a finite element method, the analysis in [7, section 5.5] allows us to derive estimates for the constants $\beta$ and $\gamma$ in (3.20) and (3.22). They depend on the finiteelement-type, and also on boundary conditions and the dimension of the problem, but, as a general rule, both $\beta$ and $\gamma$ are equal to a constant close to one times $\lambda_{\max }(Q)$, where $Q$ is the pressure mass matrix; that is, $\beta$ and $\gamma$ are proportional to $h^{2}$. As, on the other hand, $\eta$ in (3.21) is still a constant independent of $h$, it follows that, in the finite element context, $\omega$ in (3.12) is proportional to $h^{-2}$, which is somehow counter intuitive for a "relaxation" parameter. But the numerical results in section 6 clearly confirm that this rule is the correct one. This is precisely the main outcome of the theoretical results in this section: they allow us to quickly identify relevant values for $\omega$, which one could have missed with just an empirical approach.

\section{Finite difference discretization on staggered grid.}

Two-dimensional case. As a first example discretization, we consider the marker-and-cell (MAC) finite difference scheme [11]. We assume that $\Omega$ is a unit square region $(0,1) \times(0,1)$ divided into a uniform grid of cells of size $h$. The discrete velocities and pressures are distributed in a staggered arrangement. That is, the 


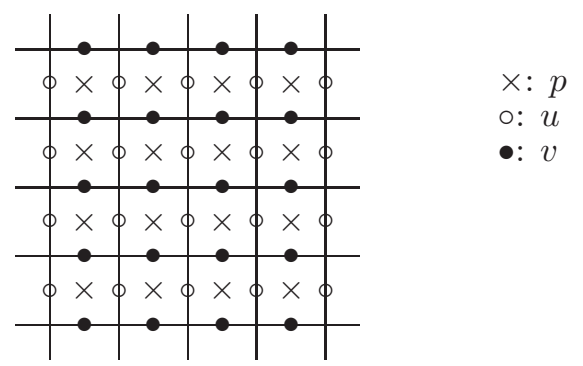

FIG. 1. Staggered grid location of unknowns for Stokes equations.

discrete pressure unknowns $p$ are defined at the cell centers (the $\times$-points), and the discrete values of $u$ and $v$, the components of the velocity vector, are located at the grid cell faces in the $\circ-$ and $\bullet-$ points, respectively; see Figure 1.

It is well known that the MAC scheme is naturally stable with $C=0$; see, for example, [15]. Hence the matrix of the discrete system is

$$
K_{h}=\left(\begin{array}{cc}
A_{h} & B_{h}^{T} \\
B_{h} & 0
\end{array}\right) .
$$

Notice that $B_{h}$ is full rank, except that $B_{h}^{T} \mathbf{1}=\mathbf{0}$, but, as discussed at the end of section 2 , this singularity entails no practical difficulty.

In order to solve the linear system, a geometric multigrid method based on the Uzawa smoother described above can be designed. Regarding the coarse-grid correction part of the algorithm, in multigrid methods for Cartesian grid discretizations, one chooses standard geometric grid coarsening, i.e., the sequence of coarse grids is obtained by doubling the mesh size in each space direction. In this framework, an appropriate coarse-grid correction consists of geometric transfer operators $R_{h, 2 h}, P_{2 h, h}$, and a direct coarse-grid discretization. The intergrid transfer operators that act on the different unknowns are dictated by the staggered grid. For the Stokes equations, they are defined as follows: At grid points corresponding to velocity unknowns $u$ and $v$, one can consider 6-point restrictions and at grid points associated with pressure unknowns $p_{h}$ a 4-point cell-centered restriction can be applied. In stencil notation these restriction operators are given by

$$
R_{h, 2 h}^{u}=\frac{1}{8}\left[\begin{array}{ccc}
1 & 2 & 1 \\
& * & \\
1 & 2 & 1
\end{array}\right], \quad R_{h, 2 h}^{v}=\frac{1}{8}\left[\begin{array}{ccc}
1 & & 1 \\
2 & * & 2 \\
1 & & 1
\end{array}\right], \quad R_{h, 2 h}^{p}=\frac{1}{4}\left[\begin{array}{ccc}
1 & & 1 \\
& * & \\
1 & & 1
\end{array}\right],
$$

respectively. For the prolongation of the corrections, we have applied the corresponding adjoint operators multiplied by a factor of 4 .

To apply the results of the preceding section, we need to set up the framework of LFA analysis. In this context, we define an extension of the staggered grid to an infinite grid $G_{h}=G_{h}^{1} \cup G_{h}^{2} \cup G_{h}^{3}$, where

$$
G_{h}^{j}:=\left\{\mathbf{x}_{k_{1}, k_{2}}^{j}=\left(k_{1}, k_{2}\right) h+\boldsymbol{\delta}^{j} \mid k_{1}, k_{2} \in \mathbb{Z}\right\} \text { with } \boldsymbol{\delta}^{j}=\left\{\begin{array}{l}
(0, h / 2) \text { if } j=1, \\
(h / 2,0) \text { if } j=2, \\
(h / 2, h / 2) \text { if } j=3 .
\end{array}\right.
$$

The velocity unknowns $u$, and $v$, are located at points $\mathbf{x}_{k_{1}, k_{2}}^{1} \in G_{h}^{1}$ and $\mathbf{x}_{k_{1}, k_{2}}^{2} \in G_{h}^{2}$, respectively, whereas pressure unknowns are situated at nodes $\mathbf{x}_{k_{1}, k_{2}}^{3} \in G_{h}^{3}$. From the 
definition of the occurring operators on $G_{h}$, the discrete solution, its current approximation, and the corresponding error or residual can be represented by formal linear combinations of complex exponential functions, the Fourier modes. These functions form a unitary basis of the space of bounded infinite grid functions, and in $G_{h}$ they are given by

$$
\boldsymbol{\varphi}_{h}\left(\boldsymbol{\theta}, \mathbf{x}_{k_{1}, k_{2}}\right):=\left(\begin{array}{c}
e^{\boldsymbol{\imath} \boldsymbol{\theta} \cdot \mathbf{x}_{k_{1}, k_{2}}^{1} / h} \\
e^{\boldsymbol{\imath} \cdot \mathbf{x}_{k_{1}, k_{2}}^{2} / h} \\
e^{\imath \boldsymbol{\theta} \cdot \mathbf{x}_{k_{1}, k_{2}}^{3} / h}
\end{array}\right),
$$

where $\boldsymbol{\theta} \in \mathbf{\Theta}:=(-\pi, \pi]^{2}, \mathbf{x}_{k_{1}, k_{2}}=\left(\mathbf{x}_{k_{1}, k_{2}}^{1}, \mathbf{x}_{k_{1}, k_{2}}^{2}, \mathbf{x}_{k_{1}, k_{2}}^{3}\right), \mathbf{x}_{k_{1}, k_{2}}^{j} \in G_{h}^{j}$. In this way, the Fourier space is defined by

$$
\mathcal{F}\left(G_{h}\right):=\operatorname{span}\left\{\boldsymbol{\varphi}_{h}(\boldsymbol{\theta}, \cdot) \mid \boldsymbol{\theta} \in \boldsymbol{\Theta}\right\},
$$

and the behavior of the multigrid components can be analyzed by evaluating their effect on the Fourier modes. The subset of low frequencies is defined as $\boldsymbol{\Theta}_{\text {low }}^{2 h}=$ $(-\pi / 2, \pi / 2]^{2}$, and the subset of high frequencies is $\Theta \backslash \Theta_{\text {low }}^{2 h}$. In the transition from $G_{h}$ to $G_{2 h}$, each low frequency $\boldsymbol{\theta}=\boldsymbol{\theta}^{00} \in \mathbf{\Theta}_{\text {low }}^{2 h}$ is coupled with three high frequencies $\boldsymbol{\theta}^{11}, \boldsymbol{\theta}^{10}, \boldsymbol{\theta}^{01}$, given by

$$
\boldsymbol{\theta}^{i j}=\boldsymbol{\theta}^{00}-\left(i \operatorname{sign}\left(\theta_{1}\right), j \operatorname{sign}\left(\theta_{2}\right)\right) \pi, i, j=0,1 .
$$

Because of this, the Fourier space can be subdivided into the corresponding fourdimensional subspaces, known as $2 h$-harmonics:

$$
\mathcal{F}^{2 h}(\boldsymbol{\theta}):=\left\{\boldsymbol{\varphi}_{h}\left(\boldsymbol{\theta}^{00}, \cdot\right), \boldsymbol{\varphi}_{h}\left(\boldsymbol{\theta}^{11}, \cdot\right), \boldsymbol{\varphi}_{h}\left(\boldsymbol{\theta}^{10}, \cdot\right), \boldsymbol{\varphi}_{h}\left(\boldsymbol{\theta}^{01}, \cdot\right)\right\} \quad \text { with } \boldsymbol{\theta}=\boldsymbol{\theta}^{00} \in \boldsymbol{\Theta}_{\text {low }}^{2 h} .
$$

The two-grid iteration matrix reads

$$
M_{h, 2 h}=S_{h}^{\nu_{2}}\left(I_{h}-P_{2 h, h}\left(K_{2 h}\right)^{-1} R_{h, 2 h} L_{h}\right) S_{h}^{\nu_{1}},
$$

where $S_{h}=I-M_{h}^{-1} K_{h}$ is the iteration matrix associated with the smoother, and $\nu_{1}$, $\nu_{2}$ are, respectively, the number of presmoothing and postsmoothing steps. The LFA two-grid convergence factor

$$
\rho=\rho\left(M_{h, 2 h}\right)
$$

is easily computed because $M_{h, 2 h}$ is block diagonal with respect to the partitioning in $2 h$-harmonics; that is, only four coupled frequencies have to be considered at a time.

Often this convergence factor is well approximated by $\mu^{\nu_{1}+\nu_{2}}$, where $\mu$ is the smoothing factor already introduced in the preceding section, which can also be defined as

$$
\mu=\sup _{\boldsymbol{\Theta} \backslash \boldsymbol{\Theta}_{\text {low }}^{2 h}} \rho\left(S_{h}(\boldsymbol{\theta})\right)
$$

We now discuss the application of Theorems 3.1 and 3.2 to bound $\mu$ by selecting the parameter $\omega$ in the Uzawa smoother on a sensible basis. ${ }^{2}$ It is well known that

\footnotetext{
${ }^{2}$ The theorems are proved for finite-dimensional matrices only. However, if LFA matrices are infinite dimensional, they are block diagonal with respect to the partitioning in $2 h$-harmonics, and one may check that applying the theorems separately to each diagonal block yields the same result as a formal extension of these theorems to the infinite-dimensional case.
} 
TABLE 1

MAC scheme for the two-dimensional problem: LFA smoothing factor $\mu$ together with its theoretical bound $\bar{\mu}$ provided in (3.6), as a function of the parameter $\omega$ given in (3.12) for $\tau \in[1,1.6]$.

\begin{tabular}{|c|c|c|c|c|c|c|c|c|}
\hline$\xi$ & & $\tau=1$ & $\tau=1.1$ & $\tau=1.2$ & $\tau=1.3$ & $\tau=1.4$ & $\tau=1.5$ & $\tau=1.6$ \\
\hline \multirow{3}{*}{0} & $\omega$ & 1 & 1.1 & 1.2 & 1.3 & 1.4 & 1.5 & 1.6 \\
& $\bar{\mu}$ & 0.5 & 0.5 & 0.5 & 0.5 & 0.5 & 0.5 & 0.6 \\
& $\mu$ & 0.5 & 0.5 & 0.5 & 0.5 & 0.5 & 0.5 & 0.6 \\
\hline \multirow{3}{*}{$10^{5}$} & $\omega$ & 1.19 & 1.30 & 1.42 & 1.54 & 1.66 & 1.78 & 1.90 \\
& $\bar{\mu}$ & 0.36 & 0.36 & 0.36 & 0.36 & 0.36 & 0.36 & 0.42 \\
& $\mu$ & 0.36 & 0.36 & 0.36 & 0.36 & 0.36 & 0.36 & 0.42 \\
\hline
\end{tabular}

the smoothing factor of the symmetric Gauss-Seidel method for the standard 5-point discretization of Laplace operator is $\mu_{A}=0.25$ [16]. Further, for the MAC scheme, $\beta$ and $\kappa_{\beta}$ in (3.9), (3.13) are equal to one. Hence, assuming that the parameter $\omega$ has been selected according to (3.12), one has, for $\xi=0$,

$$
\bar{\mu}=\max (0.5, \tau-1) .
$$

This implies that if $\tau \leq 1.5$, the upper bound on the smoothing factor is determined by the smoother for velocities, whereas, if $\tau>1.5$, it corresponds to the smoothing factor associated with Richardson iterations for the Schur complement.

For $\xi>0$, since

$$
\lambda_{\min }\left(A_{\text {high }}^{(0)}\right) \geq \frac{2}{h^{2}}, \quad \lambda_{\max }\left(A_{\text {high }}^{(0)}\right) \leq \frac{8}{h^{2}},
$$

it follows that $\eta=1 / 8$ in formula (3.10), and $\kappa_{\eta}=4$ in (3.14), so that (because $C=0$, $\gamma=0$, and, therefore, $\kappa_{\gamma}$ is not important)

$$
\mu_{S} \leq \max \left(\tau-1,1-\tau \frac{8 \nu+\xi h^{2}}{8 \nu+4 \xi h^{2}}\right) .
$$

Notice that $\mu_{A}$ decreases with increasing $\xi$ and becomes small when $\xi$ is fairly large; then, our bound $\bar{\mu}$ on $\mu$ amounts to the above value of $\mu_{S}$.

To illustrate this numerically, we fix $h^{-1}=256$ and $\nu=1$ (observe that only the ratio $\xi / \nu$ matters), and consider two values of $\xi$, whereas the parameter $\tau$ is varied in the interval $[1,1.6]$. In Table 1 , the parameter $\omega$ given by $(3.12)$, and the theoretical bound of the smoothing factor $\bar{\mu}$ provided by (3.6), together with the exact LFA smoothing factor $\mu$ are shown.

Our bound $\bar{\mu}$ accurately matches the actual smoothing factor $\mu$. Regarding the choice of parameter $\tau$, any value $\tau \in[1,1.5]$ seems appropriate. From now on, we fix the value $\tau=1.4$. This choice is based on the robustness of the resulting method for all cases analyzed in this work, including the different discretization schemes.

With parameter $\tau$ fixed, we analyze the behavior of the proposed smoother (3.1) based on symmetric Gauss-Seidel (SGS) relaxation for $M_{A}$, and compare its performance with variants where $M_{A}$ is based on a standard (forward) Gauss-Seidel (GS) method; with two sweeps, this corresponds to the method suggested in [13], which has roughly the same cost, whereas one sweep represents a cheaper alternative. In Table 2, for different values of $\xi$ and different numbers of smoothing steps, we present the smoothing factor and the LFA two-grid convergence factor (4.2), using $\omega$ as in (3.12) with $\tau=1.4$. Results are displayed for $h^{-1}=256$ and $h^{-1}=1024$. The method suggested in this work is significantly better than the method in [13], except 
TABLE 2

$M A C$ scheme for the two-dimensional problem: results using the rule (3.12) with $\tau=1.4 ; 1$ (resp., 2) sw. stands for 1 (resp., 2) GS sweep(s) for velocities in each smoothing step; 2 sw. is the strategy in [13].

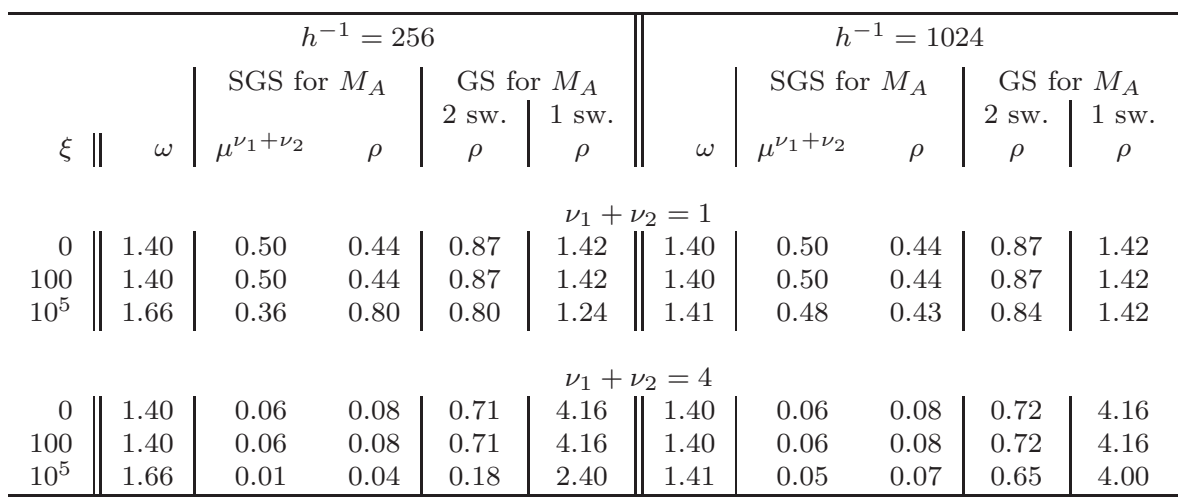

TABLE 3

$M A C$ scheme for the two-dimensional problem: number of iterations and average convergence factor for different numbers of smoothing steps.

\begin{tabular}{c|cccc|cccc}
\hline Cycle $\left(\nu_{1}, \nu_{2}\right)$ & \multicolumn{3}{|c|}{$\xi=0$} & \multicolumn{4}{c}{$\xi=10^{5}$} \\
& \multicolumn{2}{|c}{ Uzawa } & \multicolumn{2}{c}{ Vanka } & \multicolumn{2}{c}{ Uzawa } & \multicolumn{2}{c}{ Vanka } \\
& \#it. & $(\rho)$ & \#it. & $(\rho)$ & \#it. & $(\rho)$ & \#it. & $(\rho)$ \\
$\mathrm{V}(1,0)$ & - & $(-)$ & 57 & $(0.75)$ & - & - & - & - \\
$\mathrm{V}(0,1)$ & - & $(-)$ & 45 & $(0.65)$ & - & - & - & - \\
$\mathrm{W}(1,0)$ & 27 & $(0.45)$ & 26 & $(0.45)$ & 35 & $(0.53)$ & 22 & $(0.40)$ \\
& & & & & & & & \\
$\mathrm{V}(2,0)$ & 37 & $(0.57)$ & 30 & $(0.48)$ & - & - & 130 & $(0.86)$ \\
$\mathrm{V}(1,1)$ & 51 & $(0.76)$ & 31 & $(0.62)$ & - & - & 88 & $(0.84)$ \\
$\mathrm{V}(0,2)$ & 21 & $(0.33)$ & 20 & $(0.36)$ & 58 & $(0.71)$ & 115 & $(0.86)$ \\
$\mathrm{W}(1,1)$ & 17 & $(0.29)$ & 15 & $(0.24)$ & 13 & $(0.22)$ & 11 & $(0.15)$ \\
& & & & & & & & \\
$\mathrm{W}(2,1)$ & 11 & $(0.14)$ & 11 & $(0.16)$ & 9 & $(0.10)$ & 8 & $(0.08)$ \\
$\mathrm{V}(4,0)$ & 17 & $(0.27)$ & 19 & $(0.35)$ & 16 & $(0.28)$ & 12 & $(0.09)$ \\
$\mathrm{V}(2,2)$ & 22 & $(0.49)$ & 16 & $(0.31)$ & 12 & $(0.27)$ & 8 & $(0.09)$ \\
$\mathrm{V}(0,4)$ & 12 & $(0.15)$ & 13 & $(0.15)$ & 12 & $(0.15)$ & 10 & $(0.10)$ \\
$\mathrm{W}(2,2)$ & 8 & $(0.07)$ & 10 & $(0.13)$ & 8 & $(0.06)$ & 7 & $(0.04)$ \\
\hline
\end{tabular}

in one case where both perform similarly. Moreover, just one forward sweep on velocities does not lead to a convergent method. Observe further that the convergence factors associated with the proposed approach are uniformly very small when using four smoothing steps,.

We also consider the performance of multigrid cycles on finite grids. For simplicity, Dirichlet boundary conditions for the velocities are assumed (as well as in the rest of the presented numerical experiments). We compare the Uzawa smoother as defined in this work with a state-of-the-art Vanka smoother [18]. Vanka smoothers are block GS methods where one block consists of a small number of degrees of freedom. For the MAC scheme, the pressure and the velocities in the $x$ - and $y$-directions in a grid cell are simultaneously updated, resulting in $5 \times 5$ blocks. A relaxation parameter $w=0.7$ has been used in the implementation of the Vanka smoother for improving its results. In Table 3, for V-and $\mathrm{W}$-cycles and different numbers of smoothing steps, we present 
TABLE 4

MAC scheme for the three-dimensional problem: LFA smoothing factor $\mu$ together with the twogrid convergence factor $\rho$ predicted by the LFA and the $W$-cycle asymptotic convergence factor $\rho_{h}$.

\begin{tabular}{ccccc}
\hline$\nu_{1}+\nu_{2}$ & 1 & 2 & 3 & 4 \\
\hline$\mu^{\nu_{1}+\nu_{2}}$ & 0.56 & 0.32 & 0.18 & 0.10 \\
$\rho$ & 0.54 & 0.29 & 0.16 & 0.08 \\
$\rho_{h}$ & 0.51 & 0.28 & 0.15 & 0.08 \\
\hline
\end{tabular}

the number of multigrid iterations needed to reduce the residual by a factor of $10^{-10}$ in norm, on a grid with $h^{-1}=256$, together with the average convergence factor (in parentheses). Since the convergence provided by W-cycles does not depend on how the smoothing steps are distributed, we only present results for one of the possible configurations. For both smoothers, sufficiently large numbers of smoothing steps are required to obtain satisfactory $\mathrm{V}$-cycle results, and only postsmoothing appears the best choice. The $\mathrm{W}$-cycle appears faster and also more robust, converging already with one smoothing step. Regarding the comparison of both methods, the Vanka smoother appears slightly more robust when selecting V-cycles with only presmoothing. But if one focuses on the most efficient options, the multigrid performance is similar for both smoothers; that is, it does not pay off to incur the additional computational costs induced by the Vanka smoother.

Three-dimensional case. We consider the MAC discretization of (2.1) on the unit cube $\left(\Omega=(0,1)^{3}\right)$, with a uniform grid consisting of cells of size $h$. Similarly to the corresponding two-dimensional scheme, the pressure unknowns are located at the cell centers whereas the three components of the velocity are situated at the face centers. Here we consider only the Stokes case, that is, $\xi=0$.

For the solution of the corresponding linear system, we use a three-dimensional geometric multigrid method based on the Uzawa smoother, and direct coarse grid discretization. The restriction operators are dictated by the staggered location of the unknowns. We employ 12-point restrictions for the components of the velocity and an 8-point cell-centered restriction for the pressure. The prolongation operators are the corresponding adjoint operators.

The smoothing factor of the SGS method for the standard 7-point discretization of the Laplace operator is $\mu_{A}=0.3215$. Moreover, the MAC scheme is naturally stable, implying $C=0$ in (2.2), and $\beta$ and $\kappa_{\beta}$ are equal to one. Hence, taking again $\tau=1.4$ and selecting parameter $\omega$ according to (3.12) (i.e., $\omega=\tau=1.4$ ), we obtain $\mu_{S} \leq 0.4$ and, therefore, $\mu \leq \bar{\mu}=\sqrt{\mu_{A}}=0.567$. This turns out to be practically equal to the computed smoothing factor $\mu$. In Table 4, we further compare this latter with twogrid convergence factors $\rho$ predicted by LFA and the asymptotic convergence factor $\rho_{h}$ obtained on a grid with $h^{-1}=64$, using a W-cycle. As in the two-dimensional case, our theory appears relevant and the resulting method is efficient.

\section{Finite difference discretization on collocated grid.}

Two-dimensional case. The second example of discretization is a standard finite difference discretization on a collocated grid; i.e., all unknowns are located at the vertices of grid cells, which makes the discretization somewhat easier but induces the presence of spurious pressure modes with zero discrete divergence. To rule out these modes, the continuity equation is perturbed by adding an artificial elliptic pressure 
term. The matrix of the discrete system is then

$$
K=\left(\begin{array}{cc}
A & B^{T} \\
B & -C
\end{array}\right)
$$

where $C=-\alpha h^{2} \Delta_{h}$ is a scaled discrete Laplacian (with Neumann boundary conditions) acting on pressure unknowns. As this term is proportional to $h^{2}$, second order accuracy is maintained when all terms in the system are discretized with second order accuracy. Notice that $K$ is singular because $B^{T} \mathbf{1}=\mathbf{0}$ and $C \mathbf{1}=\mathbf{0}$; this singularity is managed as discussed at the end of section 2 and therefore entails no practical difficulty.

For parameter $\alpha$ a well-balanced choice needs to be made. It should be chosen sufficiently small to maintain accuracy but at the same time sufficiently large to guarantee stable solutions; here we use $\alpha=1 / 16$, which appears to be a reasonable choice in practice [12].

To solve the resulting system of algebraic equations, a geometric multigrid with standard coarse-grid correction components is adopted. Standard, $h-2 h$, grid coarsening is employed and the intergrid transfer operators are full-weighting restriction and bilinear interpolation. We analyze whether the proposed Uzawa smoother also gives satisfactory results for this type of collocated grid discretization. Basic local Fourier analysis [21] accompanies the numerical experiments, based on an infinite collocated grid $G_{h}$. Fourier modes are three-component vectors whose elements are identical to the scalar Fourier components $\varphi_{h}(\boldsymbol{\theta}, \mathbf{x})=e^{\imath \boldsymbol{\theta} \mathbf{x} / h}$.

To define $\omega$ with the rule (3.12), we need information about the parameter $\beta$ in (3.9), and, when $\xi>0$, also on $\eta$ and $\gamma$ in (3.10), (3.11). After some algebraic calculations, (3.9) is satisfied with $\beta=0.775$. For the 5 -point finite difference scheme, we have $\eta=1 / 8$ as in the preceding section, whereas, due to the simple form of the stabilization term, it is easy to derive that $\gamma=1 / 2$. Since we deal with the same 5 -point discretization of the Laplacian, the smoothing factor of the symmetric GS method remains $\mu_{A}=0.25$ when $\xi=0$ and is smaller for increasing $\xi$. Because $\kappa_{\beta}$ is larger than 1 in this example, it follows that, when using $\tau=1.4$, the dominating term in our upper bound $\bar{\mu}$ is always $\mu_{S}$; i.e., (3.6) amounts to $\mu \leq \bar{\mu}=\mu_{S}$. In Table 5 , for different values of $\xi$ and different numbers of smoothing steps, we present the

TABLE 5

Finite difference discretization on collocated grids for the two-dimensional problem: results using the rule (3.12) with $\tau=1.4 ; 1$ (resp., 2) sw. stands for 1 (resp., 2) GS sweep(s) for velocities in each smoothing step; 2 sw. is the strategy in [13].

\begin{tabular}{|c|c|c|c|c|c|c|c|c|c|c|}
\hline \multirow[b]{2}{*}{$\xi$} & \multicolumn{5}{|c|}{$h^{-1}=256$} & \multicolumn{5}{|c|}{$h^{-1}=1024$} \\
\hline & $\omega$ & \multicolumn{2}{|c|}{ SGS for $M_{A}$} & & GS for $M_{A}$ & $\omega$ & $\begin{array}{c}\text { SGS fo } \\
\mu^{\nu_{1}+\nu_{2}}\end{array}$ & $M_{A}$ & $\begin{array}{c}\text { GS } \\
2 \mathrm{sw} . \\
\rho\end{array}$ & $\begin{array}{c}M_{A} \\
1 \mathrm{sw} . \\
\rho\end{array}$ \\
\hline \multicolumn{11}{|c|}{$\nu_{1}+\nu_{2}=1$} \\
\hline 100 & 1.80 & 0.53 & 0.53 & 0.80 & 0.86 & 1.80 & 0.53 & 0.53 & 0.80 & 0.86 \\
\hline $10^{5}$ & 1.91 & 0.51 & 0.51 & 0.51 & 0.66 & 1.81 & 0.53 & 0.53 & 0.77 & 0.85 \\
\hline \multicolumn{11}{|c|}{$\nu_{1}+\nu_{2}=4$} \\
\hline 0 & 1.80 & 0.08 & 0.10 & 0.61 & 0.79 & 1.80 & 0.08 & 0.10 & 0.61 & 0.79 \\
\hline 100 & 1.80 & 0.08 & 0.10 & 0.61 & 0.79 & 1.80 & 0.08 & 0.10 & 0.61 & 0.79 \\
\hline $10^{5}$ & 1.91 & 0.06 & 0.16 & 0.16 & 0.25 & 1.81 & 0.08 & 0.09 & 0.52 & 0.73 \\
\hline
\end{tabular}


smoothing factor and the LFA two-grid convergence factor for the proposed smoother. As in the previous section, we compare with the same Uzawa smoother but with standard GS instead of SGS for $M_{A}$. Similar conclusions apply. The method proposed here exhibits significantly better convergence results than the method in [13], except for very large values of $\xi$, for which both perform similarly for $h^{-1}=256$.

As discussed at the end of section 3, it may be easier or more general to define $\omega$ based on $\beta$ from (3.20) instead of (3.9). In the present case, this would lead to a slightly larger value, namely, $\beta=1$ instead of $\beta=0.775$ considered above. Using as usual $\tau=1.4$, this yields, e.g., $\omega=1.40$ when $\xi=0$, and the two-grid convergence factor equals 0.17 for $h^{-1}=256$ and $\nu_{1}+\nu_{2}=4$. Hence there is no significant difference with $\omega$ induced by $\beta=0.775$. Similar conclusions are obtained for other values of $\xi$ and $h$, suggesting that one may indeed define $\omega$ with $\beta$ from (3.20) without significant loss of performance.

Three-dimensional case. Here we consider the Stokes case (2.1) with $\xi=0$. As in the two-dimensional case, we use a stabilization term $C=-\alpha h^{2} \Delta_{h}$ with $\alpha=1 / 16$. The multigrid components for the solution of the linear system are the well-known vertex-centered coarse-grid correction operators.

Regarding the proposed Uzawa smoother, (3.12) amounts here to $\omega=\tau / \beta$. Since $\beta=0.887$, using $\tau=1.4$ yields $\omega=1.58$. Further, calculation shows that $\kappa_{\beta}=3.52$, and therefore the bound (3.18) is $\mu_{S} \leq 0.60$. This is larger than $\left(\mu_{A}\right)^{1 / 2}=0.567$, hence the bound (3.6) on the smoothing factor is $\bar{\mu}=0.60$. Here again, numerical computation reveals that this is an accurate prediction of the actual smoothing factor, whereas the two-grid convergence factor predicted by LFA (for one smoothing step) is also $\rho=0.60$. Finally, further computation shows that the convergence factors associated with the W-cycle also accurately match this LFA two-grid convergence factor.

6. Linear finite element discretization on triangular grid. The third example of discretization deals with a linear finite element formulation of problem (2.1) on an equilateral structured triangular mesh. We thus consider the weak formulation of (2.1).

Find $(\mathbf{u}, p) \in \mathcal{U} \times \mathcal{Q}$, such that

$$
\begin{aligned}
& \xi(\mathbf{u}, \mathbf{v})+\nu(\nabla \mathbf{u}, \nabla \mathbf{v})-(p, \nabla \cdot \mathbf{v})=(\mathbf{f}, \mathbf{v}) \quad \forall \mathbf{v} \in \mathcal{U} \\
& (\nabla \cdot \mathbf{u}, q)=0 \quad \forall q \in \mathcal{Q},
\end{aligned}
$$

where $(\cdot, \cdot)$ denotes the $L^{2}$ scalar product, $\Omega$ is a bounded domain in $\mathbb{R}^{2}, \mathcal{U}=$ $\left(H_{0}^{1}(\Omega)\right)^{2}$, and $\mathcal{Q}=L_{0}^{2}(\Omega)=\left\{q \in L^{2}(\Omega) \mid \int_{\Omega} q \mathrm{~d} \mathbf{x}=0\right\}$, i.e., $L^{2}(\Omega)$ functions which only differ by a constant are not distinguished.

Let $\mathcal{T}_{h}$ be an equilateral structured triangulation of $\Omega$, and $\mathcal{U}_{h} \subset\left(H_{0}^{1}(\Omega)\right)^{2}$, and $\mathcal{Q}_{h} \subset L_{0}^{2}(\Omega)$ be the corresponding spaces of piecewise linear functions on $\mathcal{T}_{h}$. Since the pair $\left(\mathcal{U}_{h}, \mathcal{Q}_{h}\right)$ provides an unstable finite element scheme, similarly to the case of finite differences on a collocated grid, we must add an additional term to the discrete equations for stabilization. To this end, we consider the bilinear form on $\mathcal{Q}_{h} \times \mathcal{Q}_{h}$ defined by

$$
c\left(p_{h}, q_{h}\right)=\sum_{T \in \mathcal{T}_{h}} h^{2} \int_{T} \nabla p_{h} \nabla q_{h} \mathrm{~d} \mathbf{x}
$$

where $h$ denotes grid size (i.e., the length of the edge on $T$ ). The stabilized discrete formulation of the Stokes problem in its weak form reads as follows. 
TABLE 6

P1-P1 scheme for equilateral triangular grids: results using the rule (3.12) with $\tau=1.4 ; 1$ (resp., 2) sw. stands for 1 (resp., 2) GS sweep(s) for velocities in each smoothing step; 2 sw. is the strategy in [13].

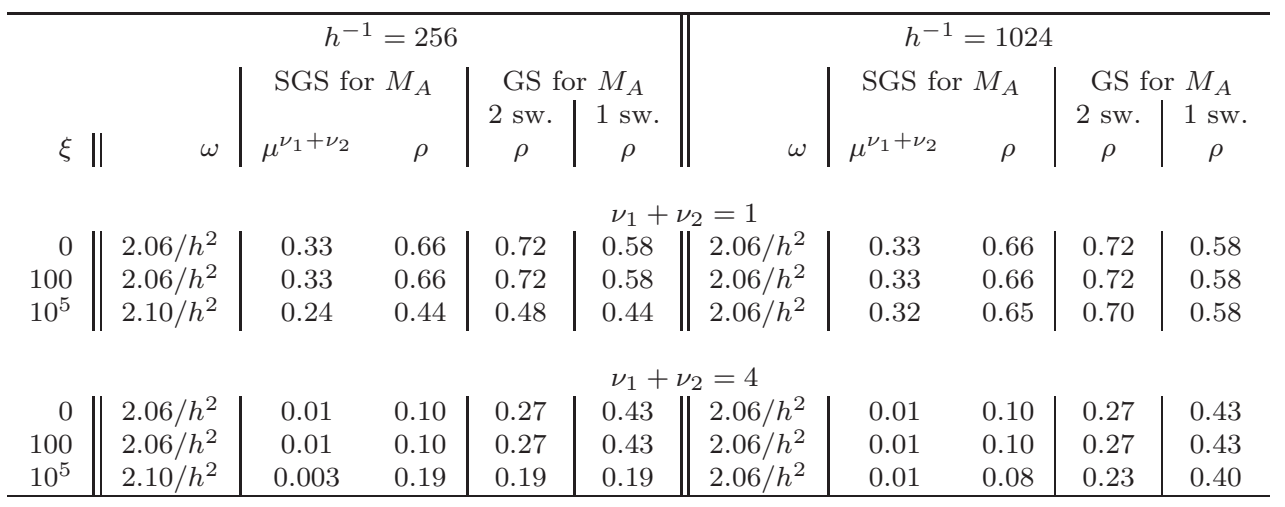

Find $\left(\mathbf{u}_{h}, p_{h}\right) \in \mathcal{U}_{h} \times \mathcal{Q}_{h}$, such that

$$
\begin{aligned}
\xi\left(\mathbf{u}_{h}, \mathbf{v}_{h}\right)+\nu\left(\nabla \mathbf{u}_{h}, \nabla \mathbf{v}_{h}\right)-\left(p_{h}, \nabla \cdot \mathbf{v}_{h}\right) & =\left(\mathbf{f}, \mathbf{v}_{h}\right) \quad \forall \mathbf{v}_{h} \in \mathcal{U}_{h} \\
\left(\nabla \cdot \mathbf{u}_{h}, q_{h}\right)+\bar{\alpha} c\left(p_{h}, q_{h}\right) & =0 \quad \forall q_{h} \in \mathcal{Q}_{h},
\end{aligned}
$$

where the term $\bar{\alpha} c\left(p_{h}, q_{h}\right)$ refers to the stabilization of the problem, and $\bar{\alpha}>0$ is a given parameter. The choice $\bar{\alpha}=1 / 12$ appears to be optimal for linear elements [6], so it is used here as well.

The discrete problem obtained gives rise to a saddle point problem like (5.1). A geometric multigrid method based on the proposed Uzawa smoother is employed to solve the discrete equations. The hierarchy of grids is obtained by a regular refinement, dividing the triangular domain into four congruent triangles, connecting the midpoints of their edges, and so forth until the mesh has the desired scale. The intergrid transfer operators are the canonical operators related to linear finite elements over triangles, i.e., the 7-point prolongation and its adjoint as the restriction [10].

LFA is typically applied to discretizations on rectangular grids; however, in [8] LFA was extended to discretizations on nonrectangular grids, in particular, to triangular grids. The key to this generalization was a two-dimensional Fourier transform using coordinates in nonorthogonal bases. A unitary basis fitting the structure of the grid is considered as the spatial basis, and its reciprocal basis is considered for the frequency space. This way, a discrete Fourier transform for discrete functions defined on nonrectangular grids can be defined and LFA on nonrectangular grids can be performed as on rectangular grids.

We first apply Theorem 3.2 to the finite element method structured equilateral triangular grid discretization. The smoothing factor of the SGS method for this discretization of the Laplacian is $\mu_{A}=0.173$. Further, we find $\eta=1 / 24$ and $\gamma=$ $\sqrt{3} h^{2} / 4$. Finally, (3.9) is satisfied with $\beta=0.68 h^{2}$.

With the help of the triangular LFA, the convergence behavior of multigrid based on the Uzawa smoother is analyzed for different values of $\xi$ and different numbers of smoothing steps. Its performance is compared to the Uzawa smoother resulting from standard GS relaxation for $M_{A}$ and the approach in [13]. These comparisons are presented in Table 6, where for different numbers of smoothing steps and different values of $\xi$ the two-grid convergence factors computed by LFA are shown, together with 
TABLE 7

Linear finite element method for the two-dimensional problem: number of iterations and asymptotic convergence factor for the $W$-cycle, with different numbers of smoothing steps.

\begin{tabular}{|c|cccc|}
\hline & \multicolumn{4}{|c|}{$\xi=0$} \\
$\left(\nu_{1}+\nu_{2}\right)$ & \#zawa & $(\rho)$ & \#it. & $(\rho)$ \\
\hline 1 & 41 & $(0.63)$ & 21 & $(0.38)$ \\
2 & 14 & $(0.22)$ & 10 & $(0.14)$ \\
3 & 10 & $(0.13)$ & 8 & $(0.08)$ \\
4 & 9 & $(0.10)$ & 7 & $(0.06)$ \\
\hline
\end{tabular}

the parameter $\omega$ given by the theory, as well as the corresponding smoothing factor. The results are displayed for $h^{-1}=256$ and $h^{-1}=1024$. For small $\xi$-values the proposed approach is superior to the other two multigrid strategies, when more than one smoothing step is considered. In the case of large $\xi$, the performance is comparable.

As in previous numerical sections, we are interested in the numerical convergence of the multigrid cycles. Moreover, we compare the behavior of the proposed strategy with multigrid based on a suitable Vanka smoother. It simultaneously updates, for each grid point, the corresponding pressure unknown and the twelve unknowns associated with the velocities located at the six points around it, resulting in $13 \times 13$ systems that are updated during the processing of a vertex of the triangulation. A relaxation parameter $w=0.7$ has been chosen for improving the smoothing properties of the Vanka smoother. In Table 7, the number of iterations to reduce the initial residual by a factor of $10^{-10}$ is shown, together with the asymptotic convergence factor in parentheses. The results are displayed for W-cycles with different numbers of smoothing steps, for $\xi=0$. The convergence of the Uzawa-based multigrid method is comparable to the performance with Vanka smoothers, that are, however, more expensive.

7. Conclusions. An Uzawa smoothing method for the multigrid solution of the generalized Stokes system has been introduced, discussed, and analyzed. It involves some parameter $\omega$, and detailed LFA smoothing analysis resulted in a formula to determine it appropriately. The resulting multigrid method appears efficient for staggered and collocated finite difference discretizations, as well as for a finite element discretization. It also compares favorably with Vanka smoother-based multigrid.

As for any study based on LFA, our analysis is restricted to constant coefficient problems. Regarding situations where, as in [9], the viscosity $\nu$ (strongly) varies inside the domain, we further observe that our rule (3.12) to define the parameter $\omega$ cannot be used anymore without ambiguity, as it sets $\omega$ proportional to $\nu$. A straightforward adaptation would then consist in letting this parameter be variable as well, to keep it proportional to $\nu$. In practice, it amounts to applying a "local" value of $\omega$ according to the "local" value of $\nu$ when relaxing the pressure unknowns as indicated in the pseudoalgorithm in section 3. Such generalization deserves, however, further investigations that are outside the scope of the present paper.

\section{REFERENCES}

[1] O. Axelsson, Iterative Solution Methods, Cambridge University Press, Cambridge, 1994.

[2] M. Benzi, G.H. Golub, And J. Liesen, Numerical solution of saddle point problems, Acta Numer., 14 (2005), pp. 1-137.

[3] A. Brandt, Multi-level adaptive solutions to boundary-value problems, Math. Comput., 31 (1977), pp. 333-390. 
[4] A. Brandt and N. Dinar, Multigrid solutions to elliptic flow problems, in Numerical Methods for Partial Differential Equations, S. Parter, ed., Academic Press, New York, 1979, pp. 53147.

[5] A. BRAndt, Rigorous quantitative analysis of multigrid I. Constant coefficients two-level cycle with L2-norm, SIAM J. Numer. Anal., 31 (1994), pp. 1695-1730.

[6] J. Donea And A. Huerta, Finite Element Methods for Flow Problems, John Wiley, Chichester, England, 2003.

[7] H. Elman, D. Silvester, And A. Wathen, Finite Elements and Fast Iterative Solvers, Oxford University Press, Oxford, 2005.

[8] F.J. Gaspar, J.L. Gracia, And F.J. Lisbona, Fourier analysis for multigrid methods on triangular grids, SIAM J. Sci. Comput., 31 (2009), pp. 2081-2102.

[9] T. Geenen, M. ur Rehman, S.P. Maclachlan, G. Segal, C. Vuik, A. P. van den Berg, AND W. SpaKman, Scalable robust solvers for unstructured FE geodynamic modeling applications: Solving the Stokes equation for models with large localized viscosity contrasts, Geochem. Geophys. Geosyst., 10 (2009).

[10] W. HackBusch, Multi-Grid Methods and Applications, Springer, Berlin, 1985.

[11] F. H. HaRlow AND J. E. Welch, Numerical calculation of time-dependent viscous incompressible flow of fluid with free surface, Phys. Fluids, 8, (1965), pp. 2182-2189.

[12] J. Linden, B. Steckel, and K. Stüben, Parallel multigrid solution of the Navier-Stokes equations on general 2D-domains, Parallel Comput., 7 (1998), pp. 461-475.

[13] J.F. Maitre, F. Musy, And P. Nigon, A fast solver for the Stokes equations using multigrid with a Uzawa smoother, in Advances in Multi-Grid Methods, Notes Numer. Fluid Mech. 11, D. Braess, W. Hackbusch, and U. Trottenberg, eds., Vieweg, Braunschweig, Germany, 1985, pp. 77-83.

[14] Y. NotAY, A new analysis of block preconditioners for saddle point problems, SIAM J. Matrix Anal. Appl., 35 (2014), pp. 143-173.

[15] D. Shin and J. Strikwerda, Inf-sup conditions for finite-difference approximations of the Stokes equations, J. Austral. Math. Soc. Ser. B, 39 (1997), pp. 121-134.

[16] K. Stüben And U. Trottenberg, Multigrid methods: Fundamental algorithms, model problem analysis and applications, in Multigrid Methods, Lecture Notes in Math. 960, W. Hackbusch and U. Trottenberg, eds., Springer-Verlag, Berlin, 1982, pp. 1-176.

[17] U. Trottenberg, C.W. Oosterlee, and A. Schüller, Multigrid, Academic Press, New York, 2001.

[18] S.P. VANKA, Block-implicit multigrid solution of Navier-Stokes equations in primitive variables, J. Comput. Phys., 65 (1986), pp. 138-158.

[19] P. Wesseling, An Introduction to Multigrid Methods, John Wiley, Chichester, UK, 1992.

[20] P. Wesseling, Principles of Computational Fluid Dynamics, Springer, Berlin, 2001.

[21] R. Wienands and W. Joppich, Practical Fourier Analysis for Multigrid Methods, Chapman and Hall/CRC Press, Boca Raton, FL, 2005.

[22] G. Wittum, Multi-grid methods for Stokes and Navier-Stokes equations with transforming smoothers: Algorithms and numerical results, Numer. Math., 54, (1989), pp. 543-563. 